Article

\title{
The Modified Helmholtz Equation on a Regular Hexagon-The Symmetric Dirichlet Problem
}

\author{
Konstantinos Kalimeris ${ }^{1, *}$ and Athanassios S. Fokas ${ }^{1,2,3}$ \\ 1 Research Center of Pure and Applied Mathematics, Academy of Athens, 11527 Athens, Greece; \\ tf227@cam.ac.uk \\ 2 Department of Applied Mathematics and Theoretical Physics, University of Cambridge, \\ Cambridge CB3 OWA, UK \\ 3 Viterbi School of Engineering, University of Southern California, Los Angeles, CA 90089-2560, USA \\ * Correspondence: kk364@cam.ac.uk or kkalimeris@academyofathens.gr
}

Received: 9 June 2020; Accepted: 27 June 2020; Published: 28 July 2020

check for updates

\begin{abstract}
Using the unified transform, also known as the Fokas method, we analyse the modified Helmholtz equation in the regular hexagon with symmetric Dirichlet boundary conditions; namely, the boundary value problem where the trace of the solution is given by the same function on each side of the hexagon. We show that if this function is odd, then this problem can be solved in closed form; numerical verification is also provided.
\end{abstract}

Keywords: unified transform; modified Helmholtz equation; global relation

\section{Introduction}

We analyse the modified Helmholtz equation in a regular hexagon using the unified transform, also known as the Fokas method. This method was introduced by one of the authors [1], for analysing integrable nonlinear partial differential equations (PDEs) [2]. Later, it was realized that it also yields novel results for linear evolution PDEs [3]; results in this direction are obtained by several authors [4-10]. Furthermore, it yields new integral representations for the solution of linear elliptic PDEs in polygonal domains [11], which in the case of simple domains can be used to obtain the analytical solution of several problems which apparently cannot be solved by the standard methods [12,13]. Recently, researchers utilised the integral representations provided by the Fokas method for the local and global wellposedness analysis of Korteweg-de Vries and nonlinear Schrödinger type PDEs [14-18], as well as for studying problems from control theory [19].

The Fokas method is based on two basic ingredients:

(1) a global relation, which is an algebraic equation that involves certain transforms of all (known and unknown) boundary values.

(2) an integral representation of the solution, which involves transforms of all boundary values.

For linear PDEs, the Fokas method involves the following:

- Given a PDE, define its formal adjoint and construct a one parameter family of solutions of this equation.

- $\quad$ By employing the given PDE and its adjoint, obtain a one parameter family of equations in conservation form. This family, together with Green's theorem, yield the global relation.

- The above family also gives rise to a certain closed differential form. The spectral analysis of this form gives rise to a scalar Riemann-Hilbert problem, which consequently yields an integral representation of the solution. This representation involves integral transforms of all the boundary 
values, and since some of them are not prescribed as boundary conditions, this form of solution is not yet effective.

- The explicit solution of the problem is derived by determining the contribution of the unknown boundary values to the integral representation. This can be achieved by using the global relation, as well as equations obtained from the global relation through certain invariant transformations.

The global relation has had important analytical and numerical implications: first, it has led to novel analytical formulations of a variety of important physical problems from water waves [20-26] to three-dimensional layer scattering [27]. Second, it has led to the development of new techniques for the Laplace, modified Helmholtz, Helmholtz, biharmonic equations, both analytical [28-35] and numerical [36-47].

The above analytical solutions are given in terms of infinite series; this is to be contrasted to other techniques based on the eigenvalues of the Laplace operator that yield the solution as a bi-infinite series. The eigenvalues of the Laplace operator for the Dirichlet, Neumann and Robin problems in the interior of an equilateral triangle were first obtained by Lamé in 1833 [48]; these results have also been derived using the Fokas method [49]. Completeness for the associated expansions for the Dirichlet and Neumann problems was obtained in [50-53] using group theoretic techniques. McCartin rederived these results $[54,55]$ and studied the connection of the eigen-structure of the equilateral triangle with that of the regular hexagon [56]. The above remarks indicate that the existing literature is based on an implicit way for deriving the solution of specific BVPs of the regular hexagon in terms of bi-infinite series. This is to be contrasted with our work which presents a direct approach for deriving explicit integral representations of the solution of a special BVP on the regular hexagon; the extension of the current methodology to more general problems is under investigation.

\section{Organisation of the Paper}

In Section 2 we implement the four steps discussed above for solving the symmetric Dirichlet problem of the modified Helmholtz equation in a regular hexagon. The main achievement of this work is presented in Section 3 and concerns the fourth step: our analysis yields the solution for the case of odd symmetric Dirichlet data in the closed form (34). We study the case of even symmetric data in Section 4, where we derive the expression (37); this expression in addition to known terms also involves an unknown term. In Section 5, Figures 1 and 2 depict the numerical verification of the main result of Section 3; also, Figures 7 and 8 indicate that the unknown term in the expression (37) is exponentially small in the high frequency limit, and hence this result provides an excellent approximation for this physically significant limit.

\section{The Basic Elements}

The equation investigated here is the modified Helmholtz equation in the interior of the regular hexagon, $D$, namely,

$$
q_{x x}+q_{y y}-4 \beta^{2} q=0, \quad(x, y) \in D,
$$

where $q(x, y)$ is a real valued function and $\beta>0$.

Using complex coordinates,

$$
z=x+i y, \quad \bar{z}=x-i y,
$$

Equation (1) becomes

$$
q_{z \bar{z}}-\beta^{2} q=0 .
$$

\subsection{The Global Relation and the Integral Representation of the Solution in the Interior of a Convex Polygon}

We first derive the global relation: 
The formal adjoint also satisfies the modified Helmholtz equation

$$
\tilde{q}_{z \bar{z}}-\beta^{2} \tilde{q}=0 .
$$

Multiplying Equation (2) by $\tilde{q}$, Equation (3) by $q$ and subtracting, we find

$$
\tilde{q} q_{z \bar{z}}-q \tilde{q}_{z \bar{z}}=0
$$

or equivalently

$$
\frac{\partial}{\partial z}\left(\tilde{q} q_{\bar{z}}-\tilde{q}_{\bar{z}} q\right)+\frac{\partial}{\partial \bar{z}}\left(q \tilde{q}_{z}-q_{z} \tilde{q}\right)=0 .
$$

Using in (5) the special solution $\tilde{q}=e^{-i \beta\left(k z-\frac{\bar{z}}{k}\right)}$ and employing Green's theorem, we obtain

$$
\int_{\partial \Omega} W(z, \bar{z}, k)=0, \quad k \in \mathbb{C},
$$

where $W$ is defined by

$$
W(z, \bar{z}, k)=e^{-i \beta\left(k z-\frac{\bar{z}}{k}\right)}\left[\left(q_{z}+i k \beta q\right) d z-\left(q_{\bar{z}}+\frac{\beta}{i k} q\right) d \bar{z}\right], \quad k \in \mathbb{C} .
$$

Suppose that $\Omega$ is the polygon defined via the points $z_{1}, z_{2}, \ldots, z_{n}, z_{n+1}=z_{1}$. Then (6) gives the following global relation for the modified Helmholtz in this polygon:

$$
\sum_{j=1}^{n} \hat{q}_{j}(k)=0, \quad k \in \mathbb{C}
$$

where $\left\{\hat{q}_{j}(k)\right\}_{1}^{n}$ are defined by

$$
\hat{q}_{j}(k)=\int_{z_{j}}^{z_{j+1}} e^{-i \beta\left(k z-\frac{\bar{z}}{k}\right)}\left[\left(q_{z}+i k \beta q\right) d z-\left(q_{\bar{z}}+\frac{\beta}{i k} q\right) d \bar{z}\right], k \in \mathbb{C},
$$

or equivalently (in local coordinates) by

$$
\begin{array}{r}
\hat{q}_{j}(k)=\int_{z_{j}}^{z_{j+1}} e^{-i \beta\left(k z-\frac{\bar{z}}{k}\right)}\left[i q_{N}^{(j)}(s)+i \beta\left(\frac{1}{k} \frac{d \bar{z}}{d s}+k \frac{d z}{d s}\right) q^{(j)}(s)\right] d s, k \in \mathbb{C}, \\
j=1, \ldots, n .
\end{array}
$$

In Equation (10) we have used the identity

$$
q_{z} d z-q_{\bar{z}} d \bar{z}=i q_{N} d s
$$

where $s$ is the arclength on the boundary $z(s)=x(s)+i y(s)$ of the polygon and $q_{N}$ denotes the derivative in the outward normal direction to the boundary of the polygon.

In order to derive the integral representation of the solution one has to implement the spectral analysis of the differential form

$$
d\left[e^{-i \beta\left(k z-\frac{\bar{z}}{k}\right)} \mu(z, k)\right]=W(z, \bar{z}, k), \quad k \in \mathbb{C} .
$$

This procedure yields the following theorem, proven in [6]:

Theorem 1. Let $\Omega$ be the interior of a convex closed polygon in the complex z-plane, with corners $z_{1}, \ldots, z_{n}, z_{n+1} \equiv z_{1}$. Assume that there exists a solution $q(z, \bar{z})$ of the modified Helmholtz equation, i.e., of 
Equation (2) with $\beta>0$, valid on $\Omega$, and suppose that this solution has sufficient smoothness on the boundary of the polygon.

Then, $q$ can be expressed in the form

$$
q(z, \bar{z})=\frac{1}{4 \pi i} \sum_{j=1}^{n} \int_{l_{j}} e^{i \beta\left(k z-\frac{\bar{z}}{k}\right)} \hat{q}_{j}(k) \frac{d k}{k}
$$

where $\left\{\hat{q}_{j}(k)\right\}_{1}^{n}$ are defined by (10), and $\left\{l_{j}\right\}_{1}^{n}$ are the rays in the complex $k$-plane

$$
l_{j}=\left\{k \in \mathbb{C}: \arg k=-\arg \left(z_{j+1}-z_{j}\right)\right\}, \quad j=1, \ldots, n
$$

oriented from zero to infinity.

Observe that the solution given in (12) is given in terms of $\left\{\hat{q}_{j}\right\}_{1}^{n}$ which involve integral transforms of both $q$ and $q_{N}$ on the boundary, i.e., both known and unknown functions.

\subsection{The Dirichlet Problem on a Regular Hexagon}

Let $D \subset \mathbb{C}$ be the interior of a regular hexagon with vertices $\left\{z_{j}\right\}_{1}^{6}$,

$$
z_{1}=\frac{l \sqrt{3}}{2}-i \frac{l}{2}=l e^{\frac{-i \pi}{6}} \quad \text { and } \quad z_{j}=\omega^{j-1} z_{1}
$$

where $l$ is the length of the side and $\omega=e^{\frac{i \pi}{3}}$. The sides $\left\{\left(z_{j}, z_{j+1}\right)\right\}_{1}^{6}, z_{7} \equiv z_{1}$ will be referred to as sides $\{(j)\}_{1}^{6}$.

For the sides $\{(j)\}_{1}^{6}$ the following parametrizations will be used:

$$
z_{1}(s)=\frac{l \sqrt{3}}{2}+i s, \quad z_{j}(s)=\left(\frac{l \sqrt{3}}{2}+i s\right) \omega^{j-1}, \quad s \in\left[-\frac{l}{2}, \frac{l}{2}\right] .
$$

The general Dirichlet problem can be uniquely decomposed to 6 simpler Dirichlet problems, by employing the decomposition

$$
q^{(j)}(s)=\sum_{i=1}^{6} \omega^{(j-1)(i-1)} g_{i}(s), \quad j=1, \ldots, 6, \quad s \in\left[-\frac{l}{2}, \frac{l}{2}\right]
$$

indeed the determinant of the matrix $\left[\left.\omega^{(j-1)(i-1)}\right|_{i, j=1, \ldots, 6}\right]$ is non-zero (Its value is $216=6^{3}$, and for the general case Det $\left.\left[\left.\omega^{(j-1)(i-1)}\right|_{i, j=1, \ldots, n}\right]=i^{\frac{2-n(n+1)}{2}} n^{n / 2}\right)$.

The existence and uniqueness of the solution of the modified Helmholtz equation shows that it is sufficient to solve each one of the above Dirichlet problems. The first of them is the symmetric Dirichlet problem, where the value $g_{1}(s)=d(s)$ is prescribed on each side. This symmetric problem is analysed in the next section.

\subsection{The Symmetric Dirichlet Problem}

The problem analysed in this subsection is the symmetric Dirichlet problem for the modified Helmholtz equation in the regular hexagon $(\Omega \equiv D)$. Let $d(s)$ be a real function with sufficient smoothness and compatibility at the vertices of the hexagon, i.e., $d\left(\frac{l}{2}\right)=d\left(-\frac{l}{2}\right)$. We prescribe the boundary conditions

$$
q^{(j)}(s)=d(s), s \in\left[-\frac{l}{2}, \frac{l}{2}\right], j=1, \ldots, 6 .
$$


The above 'symmetry' property also holds for the Neumann boundary values. This fact is the consequence of the following three observations:

- The modified Helmholtz operator $\left(\frac{\partial^{2}}{\partial z \partial \bar{z}}-\beta^{2}\right.$ Id $)$ is invariant under the transformation $z \rightarrow \omega z$, namely under rotation of $2 \pi / 3$. Since the Dirichlet data are invariant under this rotation, then the (unique) solution $q(z, \bar{z})$ of the Helmholtz equation is also invariant under this rotation.

- If $q$ is invariant under this transformation, then the differential form $q_{z} d z$ is also invariant under the transformation $z \rightarrow \omega z$ :

$$
\frac{\partial q(z)}{\partial z} d z=\frac{\partial q(\omega z)}{\partial z} d z=\frac{\partial(\omega z)}{\partial z} \frac{\partial q(\omega z)}{\partial(\omega z)} \frac{1}{\omega} d(\omega z)=\frac{\partial q(\omega z)}{\partial(\omega z)} d(\omega z)
$$

- Evaluating the above differential form on each side we obtain

$$
q_{z} d z=\frac{1}{2}\left(\dot{q}^{(j)}(s)+i q_{N}^{(j)}(s)\right) d s=\frac{1}{2}\left(d^{\prime}(s)+i q_{N}^{(j)}(s)\right) d s,
$$

where the second equality is a direct consequence of the fact that the Dirichlet data are invariant under this rotation.

Thus,

$$
q_{N}^{(j)}(s)=u(s), s \in\left[-\frac{l}{2}, \frac{l}{2}\right], j=1, \ldots, 6 .
$$

Applying the parametrization of the regular hexagon on Equation (10) we obtain:

$$
\hat{q}_{1}(k)=\hat{q}(k), \quad \hat{q}_{j}(k)=\hat{q}\left(\omega^{j-1} k\right), \quad j=1, \ldots, 6,
$$

with

$$
\hat{q}(k)=E(-i k)[i U(k)+D(k)],
$$

where $E(k), D(k)$ and $U(k)$ are defined by

$$
\begin{aligned}
& E(k)=e^{\beta\left(k+\frac{1}{k}\right) \frac{l \sqrt{3}}{2}}, \\
& D(k)=\beta\left(\frac{1}{k}-k\right) \int_{-\frac{l}{2}}^{\frac{l}{2}} e^{\beta\left(k+\frac{1}{k}\right) s} d(s) d s, \quad k \in \mathbb{C} . \\
& U(k)=\int_{-\frac{l}{2}}^{\frac{l}{2}} e^{\beta\left(k+\frac{1}{k}\right) s} u(s) d s, \quad
\end{aligned}
$$

The function $D(k)$ is known, whereas the unknown function $U(k)$ contains the unknown Neumann boundary value $u(s)=q_{N}$.

Using (15), the global relation (8) takes the form

$$
\begin{aligned}
& E(-i k) U(k)+E(-i \omega k) U(\omega k)+E\left(-i \omega^{2} k\right) U\left(\omega^{2} k\right) \\
& +E(i k) U(-k)+E(i \omega k) U(-\omega k)+E\left(i \omega^{2} k\right) U\left(-\omega^{2} k\right)=i G(k), \quad k \in \mathbb{C},
\end{aligned}
$$

where the known function $G(k)$ is defined by

$$
G(k)=\sum_{j=1}^{6} E\left(-i \omega^{j-1} k\right) D\left(\omega^{j-1} k\right), \quad k \in \mathbb{C} .
$$


The integral representation (12) of the solution takes the form

$$
q(z, \bar{z})=\frac{1}{4 \pi i} \sum_{j=1}^{6} \int_{l_{j}} e^{i \beta\left(k z-\frac{\bar{z}}{k}\right)} E\left(-i \omega^{j-1} k\right)\left[D\left(\omega^{j-1} k\right)+i U\left(\omega^{j-1} k\right)\right] \frac{d k}{k},
$$

where $\left\{l_{j}\right\}_{1}^{6}$ are the rays defined by

$$
l_{j}=\left\{k \in \mathbb{C}: \arg k=\frac{11-2 j}{6} \pi\right\}, \quad j=1, \ldots, 6,
$$

oriented from zero to infinity. The principal arguments of $\left\{l_{1}, l_{2}, l_{3}, l_{4}, l_{5}, l_{6}\right\}$ are $\left\{\frac{3 \pi}{2}, \frac{7 \pi}{6}, \frac{5 \pi}{6}, \frac{\pi}{2}, \frac{\pi}{6}, \frac{11 \pi}{6}\right\}$, respectively.

Since the function $d(s)$ can be uniquely written as a sum of an odd and an even function, we will only consider two particular cases:

(i) the odd case, $d(-s)=-d(s)$;

(ii) the even case $d(-s)=d(s)$.

The solution and the Neumann boundary values inherit the analogous properties:

(i) in the odd case, $u(-s)=-u(s)$, which yields $U(-k)=-U(k)$;

(ii) in the even case, $u(-s)=u(s)$, which yields $U(-k)=U(k)$ for all $k \in \mathbb{C}$.

\section{Derivation of the Solution for the Symmetric Odd Case}

In what follows we will show that the contribution of the unknown functions $\left\{U\left(\omega^{j-1} k\right)\right\}_{1}^{6}$ to the solution representation (19) can be computed explicitly.

Applying the condition $U(-k)=-U(k)$ in (17) we obtain the equation

$$
\Delta(i k) U(k)+\Delta(i \omega k) U(\omega k)+\Delta\left(i \omega^{2} k\right) U\left(\omega^{2} k\right)=-i G(k), \quad k \in \mathbb{C},
$$

where $G(k)$ is given in (18) and $\Delta(k)$ is defined by

$$
\Delta(k)=E(k)-E(-k)
$$

Solving (21) for $U(k)$ and substituting the resulting expression in (15) we find

$$
\begin{aligned}
\hat{q}(k) & =E(-i k) D(k)+\frac{E(-i k) G(k)}{\Delta(i k)} \\
& +i[E(-i k) E(-i \omega k)-E(-i k) E(i \omega k)] \frac{U(\omega k)}{\Delta(i k)} \\
& +i\left[E(-i k) E\left(-i \omega^{2} k\right)-E(-i k) E\left(i \omega^{2} k\right)\right] \frac{U\left(\omega^{2} k\right)}{\Delta(i k)}
\end{aligned}
$$

The functions $\hat{q}_{j}(k)$ can be obtained from (22) by replacing $k$ with $\omega^{j-1} k$ for $j=1, \ldots, 6$.

Regarding the integral representation of the solution, we restrict our attention to the first integral of (19), namely the integral along $l_{1}$ (the negative imaginary axis).

Let

$$
\mathcal{P}=e^{i \beta(k z-\bar{z})} .
$$

Solving (21) for $U(k)$ and substituting the resulting expression in the first integral of (19) we find that the known part of this integral is given by the expression

$$
F_{1}=\frac{1}{4 \pi i} \int_{l_{1}} \mathcal{P} E(-i k)\left[D(k)+\frac{G(k)}{\Delta(i k)}\right] \frac{d k}{k} .
$$


The unknown part involves the functions $U(\omega k)$ and $U\left(\omega^{2} k\right)$ and is given by

$$
\begin{aligned}
C_{1} & =\frac{1}{4 \pi} \int_{l_{1}} \mathcal{P}\left[E(-i k) E(-i \omega k) \frac{U(\omega k)}{\Delta(i k)}+E(-i k) E\left(-i \omega^{2} k\right) \frac{U\left(\omega^{2} k\right)}{\Delta(i k)}\right] \frac{d k}{k} \\
& -\frac{1}{4 \pi} \int_{l_{1}} \mathcal{P}\left[E(-i k) E(i \omega k) \frac{U(\omega k)}{\Delta(i k)}+E(-i k) E\left(i \omega^{2} k\right) \frac{U\left(\omega^{2} k\right)}{\Delta(i k)}\right] \frac{d k}{k} .
\end{aligned}
$$

In what follows we will show that the contribution of the unknown functions, namely of the sum $\Sigma_{1}^{6} C_{j}$, can be computed in terms of the given boundary conditions.

The first integral in the rhs of $C_{1}$ can be deformed from $l_{1}$ to $l_{1}^{\prime}$, where $l_{1}^{\prime}$ is a ray with $\frac{7 \pi}{6} \leq \arg k \leq$ $\frac{3 \pi}{2}$; choosing $l_{1}^{\prime} \equiv l_{2}$ we obtain

$$
C_{1}=\hat{C}_{1}+\check{C}_{1}
$$

where

$$
\hat{C}_{1}=\frac{1}{4 \pi} \int_{l_{2}} \mathcal{P}\left[E(-i k) E(-i \omega k) \frac{U(\omega k)}{\Delta(i k)}+E(-i k) E\left(-i \omega^{2} k\right) \frac{U\left(\omega^{2} k\right)}{\Delta(i k)}\right] \frac{d k}{k}
$$

and

$$
\check{C}_{1}=-\frac{1}{4 \pi} \int_{l_{1}} \mathcal{P}\left[E(-i k) E(i \omega k) \frac{U(\omega k)}{\Delta(i k)}+E(-i k) E\left(i \omega^{2} k\right) \frac{U\left(\omega^{2} k\right)}{\Delta(i k)}\right] \frac{d k}{k} .
$$

The above deformation is justified, since it can be shown that the integrand of $\hat{C}_{1}$ is bounded and analytic in the region where $\arg k \in\left[\frac{7 \pi}{6}, \frac{3 \pi}{2}\right]$ : letting $a=e^{i \frac{\pi}{6}}$, we can rewrite the first term of the integrand of $\hat{C}_{1}$ in the form

$$
\mathcal{P} E^{-\frac{2}{\sqrt{3}}}(i a k) \frac{E^{\frac{2}{\sqrt{3}}}(i a k) E(-i k) E(-i \omega k) E^{\frac{1}{\sqrt{3}}}(\omega k)}{\Delta(i k)} E^{-\frac{1}{\sqrt{3}}}(\omega k) U(\omega k) .
$$

We observe the following:

- $\quad$ The zeros of $\Delta(i k)$ occur when $i k+\frac{1}{i k} \in e^{-i \frac{\pi}{2}} \mathbb{R}$, thus $k \in \mathbb{R}$.

- The function $\mathcal{P} E^{-\frac{2}{\sqrt{3}}}(i a k)=e^{i \beta k\left(z-z_{2}\right)+\frac{\beta}{i k}\left(\bar{z}-\bar{z}_{2}\right)}$ is bounded and analytic for $\arg k \in\left[\frac{7 \pi}{6}, \frac{3 \pi}{2}\right]$.

Indeed, if $z \in D$, then $\frac{5 \pi}{6} \leq \arg \left(z-z_{2}\right) \leq \frac{3 \pi}{2}$. Thus, if $\frac{7 \pi}{6} \leq \arg k \leq \frac{3 \pi}{2}$, it follows that $2 \pi \leq \arg \left[k\left(z-z_{2}\right)\right] \leq 3 \pi$. Hence, $\operatorname{Re}\left\{i k\left(z-z_{2}\right)\right\} \leq 0$.

Therefore, the exponentials $e^{i \beta k\left(z-z_{2}\right)}$ and $e^{\frac{\beta}{i k}\left(\bar{z}-\bar{z}_{2}\right)}$ are bounded.

- The function $E^{-\frac{1}{\sqrt{3}}}(\omega k) U(\omega k)$ is bounded and analytic for $\arg k \in\left[\frac{7 \pi}{6}, \frac{13 \pi}{6}\right]$, namely in the region where $\operatorname{Re}(\omega k) \geq 0$.

Indeed, this expression involves the exponentials $e^{\beta \omega k\left(s-\frac{l}{2}\right)}$ and $e^{\beta \frac{1}{\omega k}\left(s-\frac{l}{2}\right)}$, which are bounded in this region, since $s \leq \frac{l}{2}$.

- The function

$$
\frac{E^{\frac{2}{\sqrt{3}}}(i a k) E(-i k) E(-i \omega k) E^{\frac{1}{\sqrt{3}}}(\omega k)}{\Delta(i k)}=\frac{E^{\frac{1}{\sqrt{3}}}(k)}{\Delta(i k)}
$$

is bounded and analytic for $\arg k \in\left[\frac{7 \pi}{6}, \frac{3 \pi}{2}\right]$.

Indeed, since $k$ is at the lower half plane, then

$$
\frac{E^{\frac{1}{\sqrt{3}}}(k)}{\Delta(i k)} \sim \frac{E^{\frac{1}{\sqrt{3}}}(k)}{E(i k)}=E^{-\frac{2}{\sqrt{3}}}\left(\omega^{2} k\right), \quad k \rightarrow \infty,
$$

which is bounded if $\operatorname{Re}\left(\omega^{2} k\right) \geq 0$.

If $\arg k \in\left[\frac{7 \pi}{6}, \frac{3 \pi}{2}\right]$, then $\arg \left(\omega^{2} k\right) \in\left[\frac{11 \pi}{6}, \frac{13 \pi}{6}\right]$, which yields $\operatorname{Re}\left(\omega^{2} k\right)>0$. 
Similar considerations apply to the second term of the integrand of $\hat{C}_{1}$; this term can be rewritten in the form

$$
\mathcal{P} E^{-\frac{2}{\sqrt{3}}}(i a k) \frac{E^{\frac{2}{\sqrt{3}}}(i a k) E(-i k) E\left(-i \omega^{2} k\right) E^{\frac{1}{\sqrt{3}}}\left(\omega^{2} k\right)}{\Delta(i k)} E^{-\frac{1}{\sqrt{3}}}\left(\omega^{2} k\right) U\left(\omega^{2} k\right) .
$$

We observe the following:

- The function $\mathcal{P} E^{-\frac{2}{\sqrt{3}}}(i a k)=e^{i \beta k\left(z-z_{2}\right)+\frac{\beta}{i k}\left(\bar{z}-\bar{z}_{2}\right)}$ is bounded and analytic for $\arg k \in\left[\frac{7 \pi}{6}, \frac{3 \pi}{2}\right]$.

- The function $E^{-\frac{1}{\sqrt{3}}}\left(\omega^{2} k\right) U\left(\omega^{2} k\right)$ is bounded and analytic for $\arg k \in\left[\frac{5 \pi}{6}, \frac{11 \pi}{6}\right]$, namely in the region where $\operatorname{Re}\left(\omega^{2} k\right) \geq 0$.

- In the lower half plane

$$
\frac{E^{\frac{2}{\sqrt{3}}}(i a k) E(-i k) E\left(-i \omega^{2} k\right) E^{\frac{1}{\sqrt{3}}}\left(\omega^{2} k\right)}{\Delta(i k)} \sim 1, \quad k \rightarrow \infty .
$$

Thus, it is bounded and analytic for $\arg k \in\left[\frac{7 \pi}{6}, \frac{3 \pi}{2}\right]$.

Using the underlined symmetries, we can express the integral representation of the solution in the form

$$
q=\sum_{j=1}^{6} F_{j}+\sum_{j=1}^{6} C_{j}=\sum_{j=1}^{6} F_{j}+\sum_{j=1}^{6}\left(\hat{C}_{j}+\check{C}_{j}\right)
$$

where $F_{j}$ and $C_{j}$ are given by applying in (23) and (24) the following rotations:

$$
k \rightarrow \omega^{j-1} k, \quad l_{1} \rightarrow l_{j}, \quad l_{2} \rightarrow l_{j+1}, \quad j=2, \ldots, 6 ; \quad l_{7}:=l_{1} .
$$

We define $\widetilde{C}_{j}=\hat{C}_{j-1}+\check{C}_{j}, j=1, \ldots, 6$, where we employ the notation $\hat{C}_{0}=\hat{C}_{6}$. Then, we rewrite the expression in (25) in the form

$$
q=\sum_{j=1}^{6} F_{j}+\sum_{j=0}^{5} \hat{C}_{j}+\sum_{j=1}^{6} \check{C}_{j}=\sum_{j=1}^{6} F_{j}+\sum_{j=1}^{6}\left(\hat{C}_{j-1}+\check{C}_{j}\right)=\sum_{j=1}^{6} F_{j}+\sum_{j=1}^{6} \widetilde{C}_{j} .
$$

Thus, it is sufficient to compute the contribution $\left\{\widetilde{\mathcal{C}}_{j}\right\}_{1}^{6}$. In this direction we find (via rotation) that

$$
\check{C}_{2}=-\frac{1}{4 \pi} \int_{l_{2}} \mathcal{P}\left[E(-i \omega k) E\left(i \omega^{2} k\right) \frac{U\left(\omega^{2} k\right)}{\Delta(i \omega k)}+E(-i \omega k) E\left(i \omega^{3} k\right) \frac{U\left(\omega^{3} k\right)}{\Delta(i \omega k)}\right] \frac{d k}{k} .
$$

Thus

$$
\begin{aligned}
\widetilde{C}_{2} & =\hat{C}_{1}+\check{C}_{2} \\
& =\frac{1}{4 \pi} \int_{l_{2}} \mathcal{P}\left[E(-i k) E(-i \omega k) \frac{U(\omega k)}{\Delta(i k)}+E(-i k) E\left(-i \omega^{2} k\right) \frac{U\left(\omega^{2} k\right)}{\Delta(i k)}\right] \frac{d k}{k} \\
& -\frac{1}{4 \pi} \int_{l_{2}} \mathcal{P}\left[E(-i \omega k) E\left(i \omega^{2} k\right) \frac{U\left(\omega^{2} k\right)}{\Delta(i \omega k)}+E(-i \omega k) E\left(i \omega^{3} k\right) \frac{U\left(\omega^{3} k\right)}{\Delta(i \omega k)}\right] \frac{d k}{k}
\end{aligned}
$$

Using that $\omega^{3}=-1$ and $U(-k)=-U(k)$ the above expression is simplified to

$$
\widetilde{C}_{2}=\frac{1}{4 \pi} \int_{l_{2}} \mathcal{P} E(-i k) E(-i \omega k) \frac{\Delta(i k) U(k)+\Delta(i \omega k) U(\omega k)+\Delta\left(i \omega^{2} k\right) U\left(\omega^{2} k\right)}{\Delta(i k) \Delta(i \omega k)} \frac{d k}{k} .
$$

Employing the global relation (21) we obtain

$$
\widetilde{C}_{2}=\frac{1}{4 \pi i} \int_{l_{2}} \mathcal{P} E(-i k) E(-i \omega k) \frac{G(k)}{\Delta(i k) \Delta(i \omega k)} \frac{d k}{k} .
$$


In summary, the solution takes the form

$$
q=\sum_{j=1}^{6} F_{j}+\sum_{j=1}^{6} \widetilde{C}_{j}
$$

where $F_{j}$ is defined by

$$
F_{j}=\frac{1}{4 \pi i} \int_{l_{j}} \mathcal{P} E\left(-i \omega^{j-1} k\right)\left[D\left(\omega^{j-1} k\right)+\frac{G\left(\omega^{j-1} k\right)}{\Delta\left(i \omega^{j-1} k\right)}\right] \frac{d k}{k}
$$

and $\widetilde{C}_{j}$ is defined by

$$
\widetilde{C}_{j}=\frac{1}{4 \pi i} \int_{l_{j}} \mathcal{P} E\left(-i \omega^{j-2} k\right) E\left(-i \omega^{j-1} k\right) \frac{G\left(\omega^{j-2} k\right)}{\Delta\left(i \omega^{j-1} k\right) \Delta\left(i \omega^{j-2} k\right)} \frac{d k}{k} .
$$

Note also that the integrals of $\widetilde{C}_{j}$ can be deformed on a sector of angle $\frac{2 \pi}{3}$. For example, in $\widetilde{C}_{2}$ the ray $l_{2}$ can be deformed in a ray $l_{2}^{\prime}$ in the sector $\arg k \in\left(\pi, \frac{5 \pi}{3}\right)$; analogous results are valid for the remaining $\left\{\widetilde{C}_{j}\right\}_{1}^{6}$.

Observing that $G(\omega k)=G(k)$, Equation (29) can be further simplified to

$$
q=\frac{1}{4 \pi i} \sum_{j=1}^{6} \int_{l_{j}} \mathcal{P}\left[E\left(-i \omega^{j-1} k\right) D\left(\omega^{j-1} k\right)+\frac{E\left(-i \omega^{j} k\right) G\left(\omega^{j-1} k\right)}{\Delta\left(i \omega^{j-1} k\right) \Delta\left(i \omega^{j-2} k\right)}\right] \frac{d k}{k} .
$$

In order to write the integral representation in a more compact form we make the change of variables $k \rightarrow \omega^{1-j} k$ in the integrals in $F_{j}$ and $\widetilde{C}_{j}$. In this procedure:

1. the fraction $\frac{d k}{k}$ remains invariant;

2. the rays $l_{j}$ become $l_{1}$;

3. the exponent $\mathcal{P}=e^{i \beta\left(k z-\frac{\bar{z}}{k}\right)}$ becomes $e^{i \beta\left(\omega^{1-j} k z-\frac{\bar{z}}{\omega^{1-j_{k}}}\right)}$;

4. the remaining integrands are equal to the corresponding integrands in $F_{1}$ and $\widetilde{C}_{1}$.

Thus, we obtain

$$
q=\frac{1}{4 \pi i} \int_{l_{1}} \mathcal{T}\left[E(-i k) D(k)-\frac{E(-i \omega k)}{\Delta(i k) \Delta\left(i \omega^{2} k\right)} G(k)\right] \frac{d k}{k} .
$$

where

$$
\mathcal{T}=\sum_{j=1}^{6} e^{i \beta\left(\omega^{1-j} k z-\frac{\bar{z}}{\omega^{1-j_{k}}}\right)} .
$$

We make the change of variables $k \rightarrow-i k$ in the integrand of (33), so that the contour of integration transforms from the negative imaginary axis $l_{1}$ to the real imaginary axis, and we summarize the above result in the form of a proposition.

Proposition 1. Let $q$ satisfy the modified Helmholtz Equation (2) in the interior of a regular hexagon defined in (13). Assume that on each side of this hexagon an odd symmetric Dirichlet boundary condition is prescribed, namely,

$$
q^{(j)}(s)=d(s), \quad s \in\left[-\frac{l}{2}, \frac{l}{2}\right], j=1, \ldots, 6,
$$

with $d(-s)=-d(s)$ and $d\left(-\frac{l}{2}\right)=d\left(\frac{l}{2}\right)=0$. 
The solution q can be computed in closed form:

$$
q(z, \bar{z})=\frac{1}{4 \pi i} \int_{0}^{\infty} R(k, z, \bar{z})\left[E(-k) D(-i k)-\frac{E(-\omega k)}{\Delta(k) \Delta\left(\omega^{2} k\right)} G(-i k)\right] \frac{d k}{k},
$$

where $R(k, z, \bar{z}), D(k), E(k), G(k), \Delta(k)$ are defined as follows:

$$
\begin{aligned}
& R(k, z, \bar{z})=\sum_{j=1}^{6} e^{\beta\left(\omega^{1-j} k z+\frac{\bar{z}}{\omega^{1-j} k}\right)} \\
& E(k)=e^{\beta\left(k+\frac{1}{k}\right) \frac{1 \sqrt{3}}{2}}, \quad D(k)=\beta\left(\frac{1}{k}-k\right) \int_{-\frac{1}{2}}^{\frac{l}{2}} e^{\beta\left(k+\frac{1}{k}\right) s} d(s) d s, \\
& G(k)=\sum_{j=1}^{6} E\left(-i \omega^{j-1} k\right) D\left(\omega^{j-1} k\right), \quad \Delta(k)=E(k)-E(-k), \quad k \in \mathbb{C} .
\end{aligned}
$$

\section{The Symmetric Even Case}

Applying the condition $U(-k)=U(k)$ in (17) we obtain the following equation

$$
\Delta^{+}(i k) U(k)+\Delta^{+}(i \omega k) U(\omega k)+\Delta^{+}\left(i \omega^{2} k\right) U\left(\omega^{2} k\right)=i G(k), \quad k \in \mathbb{C},
$$

where

$$
\Delta^{+}(k)=E(k)+E(-k)
$$

and $G(k)$ is known and given in (18).

Following the same stems used in Section 3 we derive the analogue of (28), which yields the following formula for $\widetilde{C}_{2}$ :

$$
\begin{aligned}
\widetilde{C}_{2} & =\frac{1}{4 i \pi} \int_{l_{2}} \mathcal{P} E(-i k) E(-i \omega k) \frac{G(k)}{\Delta^{+}(i k) \Delta^{+}(i \omega k)} \frac{d k}{k} \\
& +\frac{1}{2 \pi} \int_{l_{2}} \mathcal{P} \frac{U\left(\omega^{2} k\right)}{\Delta^{+}(i k) \Delta^{+}(i \omega k)} \frac{d k}{k}
\end{aligned}
$$

where in addition to the known part which involves $G(k)$, there now exists an unknown part which involves $U\left(\omega^{2} k\right)$

Thus, the analogue of (29) now takes the form

$$
q=\sum_{j=1}^{6} F_{j}+\sum_{j=1}^{6} A_{j}+\sum_{j=1}^{6} B_{j}
$$

where $F_{j}$ is known function defined by

$$
F_{j}=\frac{1}{4 \pi i} \int_{l_{j}} \mathcal{P} E\left(-i \omega^{j-1} k\right)\left[D\left(\omega^{j-1} k\right)+\frac{G\left(\omega^{j-1} k\right)}{\Delta^{+}\left(i \omega^{j-1} k\right)}\right] \frac{d k}{k},
$$

$A_{j}$ is also known and defined by

$$
A_{j}=\frac{1}{4 \pi i} \int_{l_{j}} \mathcal{P} E\left(-i \omega^{j-2} k\right) E\left(-i \omega^{j-1} k\right) \frac{G\left(\omega^{j-2} k\right)}{\Delta^{+}\left(i \omega^{j-1} k\right) \Delta^{+}\left(i \omega^{j-2} k\right)} \frac{d k}{k},
$$

whereas $B_{j}$ is the unknown function defined by

$$
B_{j}=\frac{1}{2 \pi} \int_{l_{j}} \mathcal{P} \frac{U\left(\omega^{j} k\right)}{\Delta^{+}\left(i \omega^{j-1} k\right) \Delta^{+}\left(i \omega^{j-2} k\right)} \frac{d k}{k} .
$$


It can be shown that each of $B_{j}$ decays exponentially fast as $\beta \rightarrow \infty$. The rigorous proof of this statement will be presented elsewhere. In the next section, this fact will be indicated via the numerical evaluation of each of the terms appearing in Equation (37).

\section{Illustration of the Results}

\subsection{Odd Case}

Below we depict the solution obtained by (34) for various choices of the Dirichlet datum $d(s)$ and of the parameter $\beta$. At all the examples we have fixed the length of the side of the hexagon $l=2$.

For the first example we employ the Dirichlet datum $d(s)=\sin (\pi s)$ and the parameter $\beta=1$; see Figure 1.

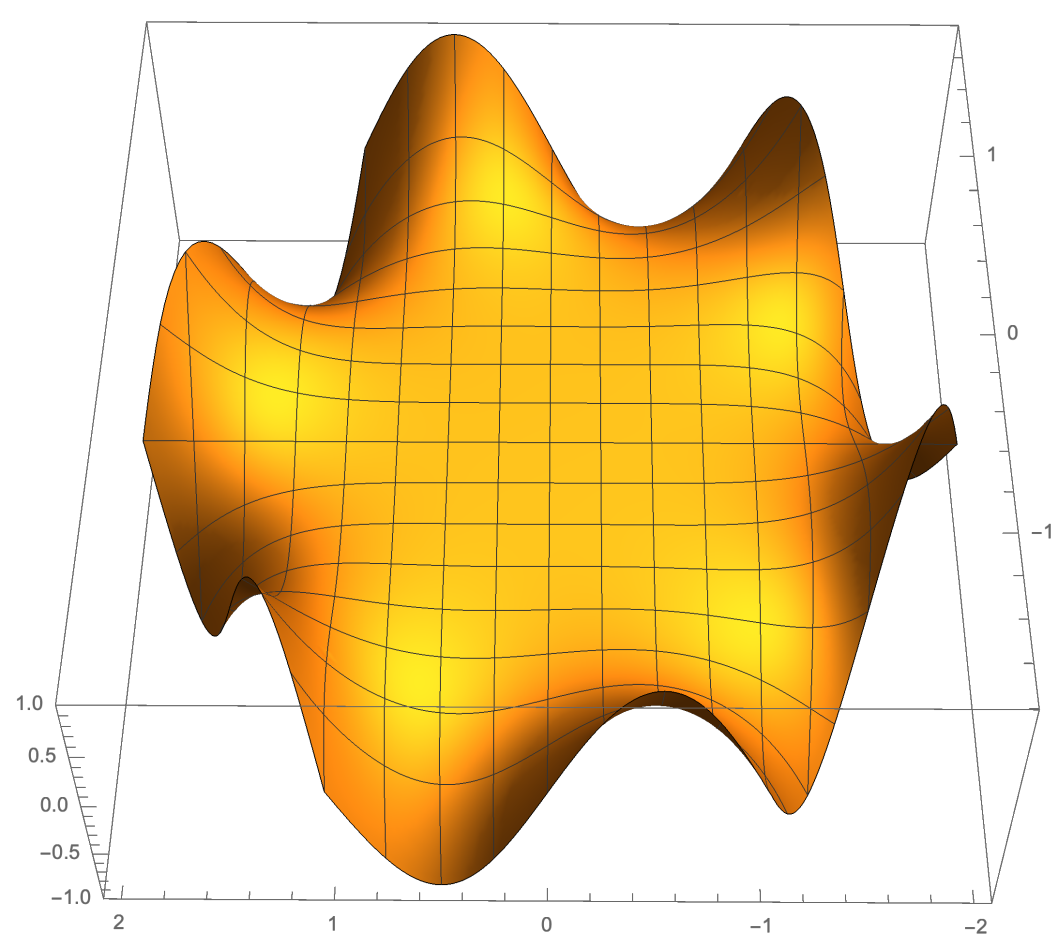

Figure 1. The solution $q$ given by (34) for $d(s)=\sin (\pi s), l=2$ and $\beta=1$.

We also depict the deviation of $d(s)$ from the function obtained by the integral representation (34) evaluated at the side of the hexagon, namely at $x=\frac{l \sqrt{3}}{2}=\sqrt{3}$ and $y=s \in\left[-\frac{l}{2}, \frac{l}{2}\right] \equiv[-1,1]$; see Figure 2. 


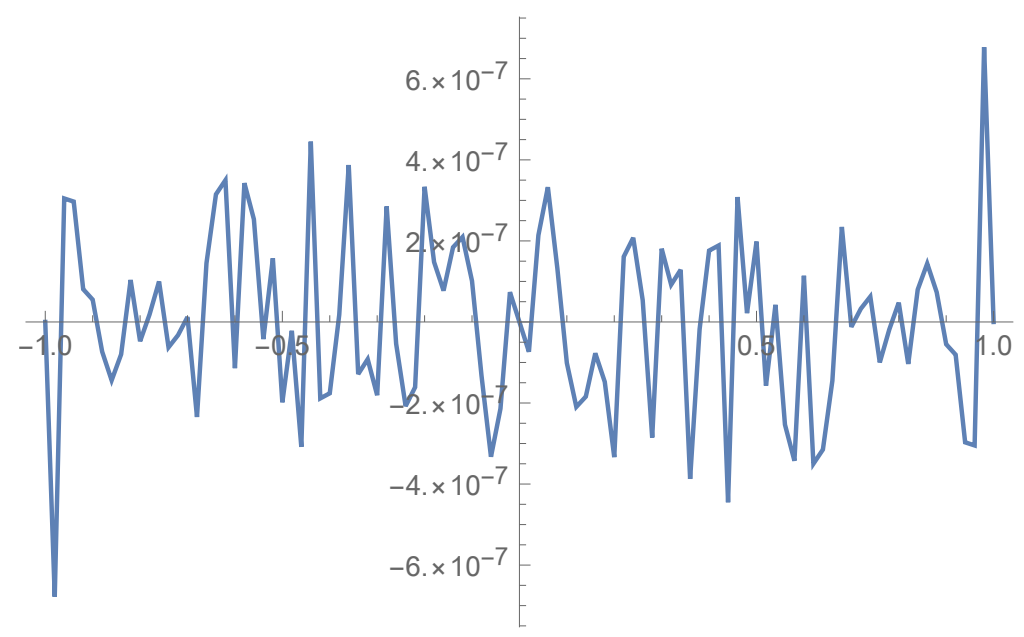

Figure 2. The deviation of $q$ (given by (34)) from the actual Dirichlet datum $d(s)$ evaluated at the side of the hexagon; here we employ $d(s)=\sin (\pi s), l=2$ and $\beta=1$.

For the second example we employ the Dirichlet datum $d(s)=\sin (\pi s)$ and the parameter $\beta=1 / 5$; see Figure 3 .

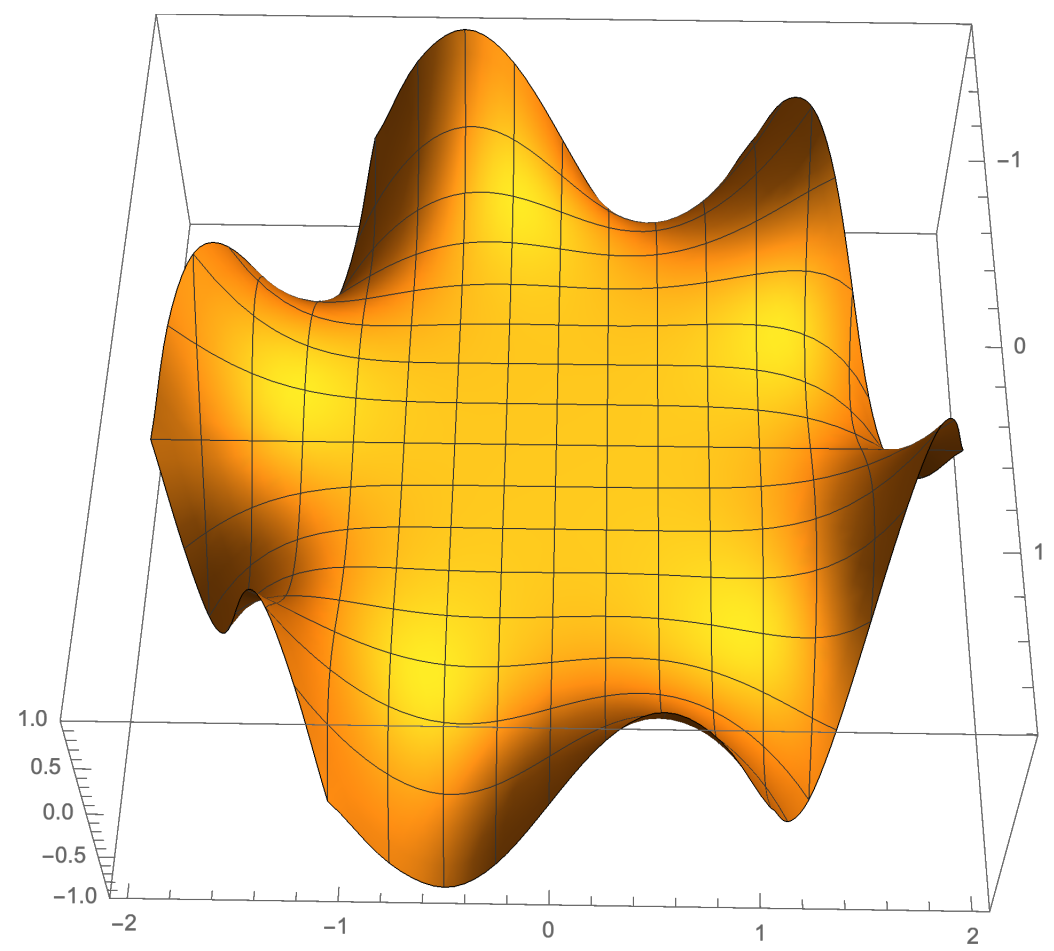

Figure 3. The solution $q$ given by (34) for $d(s)=\sin (\pi s), l=2$ and $\beta=1 / 5$.

For the third example we employ the Dirichlet datum $d(s)=\sin (2 \pi s)$ and the parameter $\beta=1$; see Figure 4. 


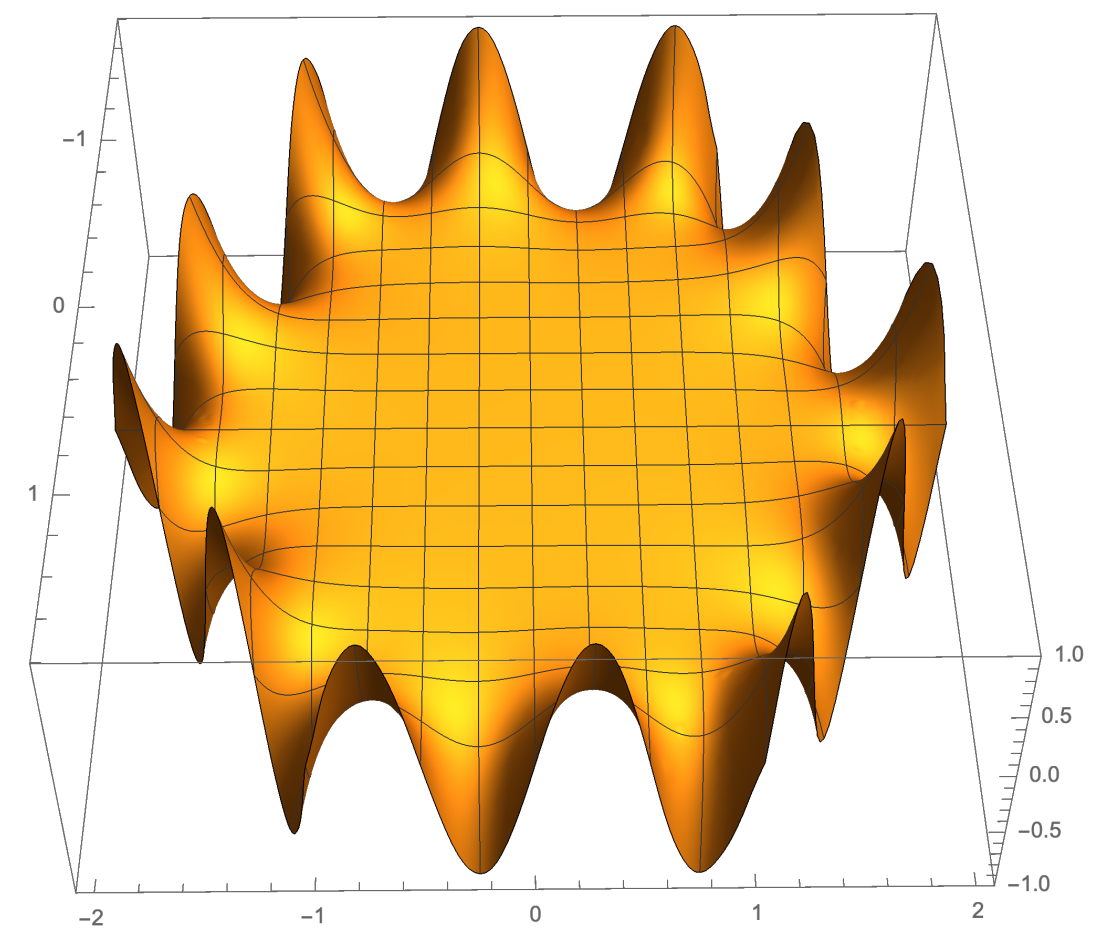

Figure 4 . The solution $q$ given by (34) for $d(s)=\sin (2 \pi s), l=2$ and $\beta=1$.

\subsection{Even Case}

In this case we employ the Dirichlet datum $d(s)=\cos \left(\frac{\pi}{2} s\right)$ and the parameter $\beta=1$ at the known part of the rhs of the formula (37), namely the expression

$$
\sum_{j=1}^{6} F_{j}+\sum_{j=1}^{6} A_{j}
$$

where $F_{j}$ and $A_{j}$ are given by (38) and (39), respectively; see Figure 5. 


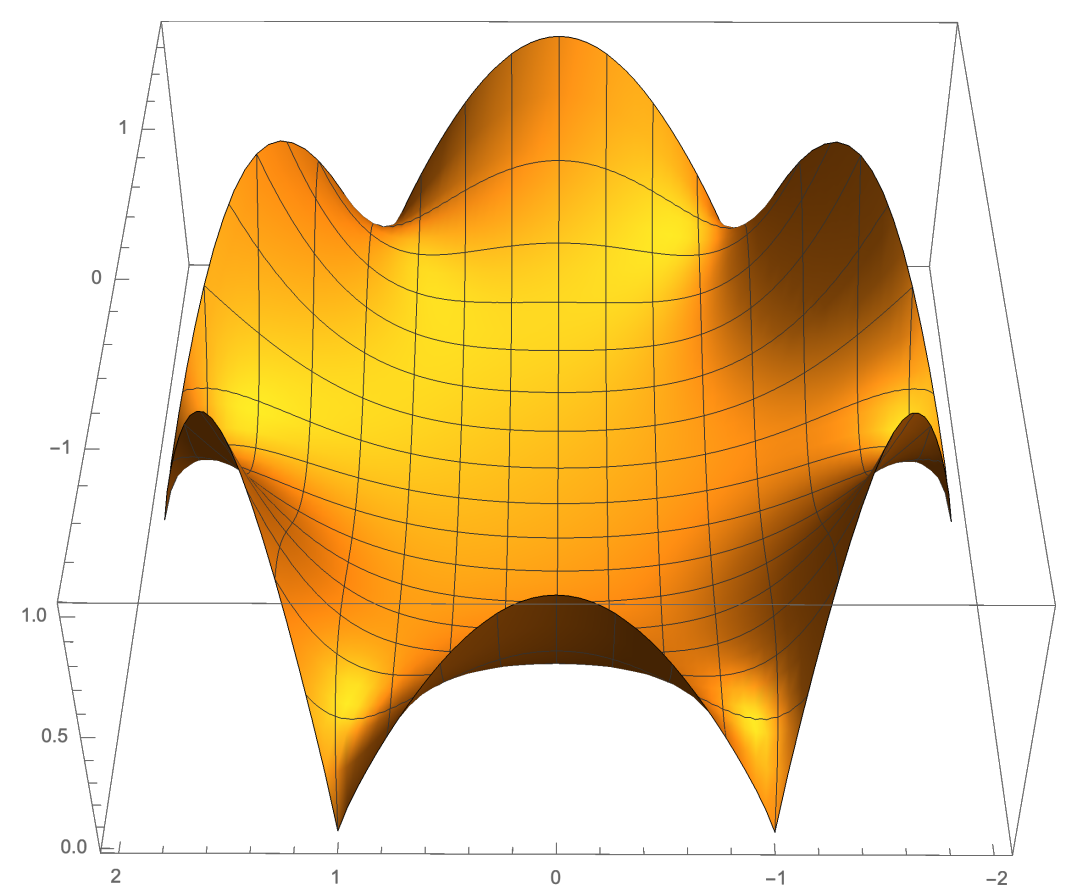

Figure 5. The known part of the solution $q$ given by (41) for $d(s)=\cos \left(\frac{\pi}{2} s\right), l=2$ and $\beta=1$.

We also depict the deviation of $d(s)$ from the above expression evaluated at the side of the hexagon, namely at $x=\sqrt{3}$ and $y=s \in[-1,1]$. This is equal to the contribution $\sum_{j=1}^{6} B_{j}$, with $B_{j}$ given by (40); see Figure 6.

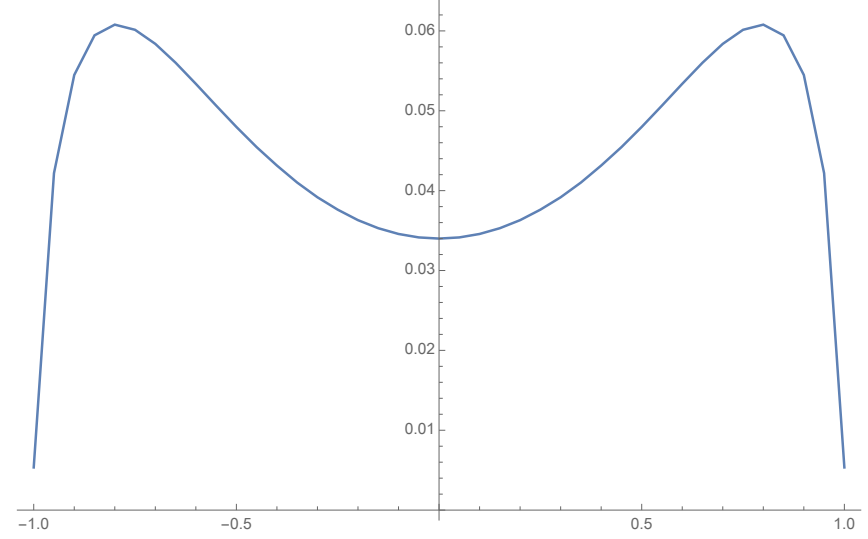

Figure 6. The deviation of the known part of the solution $q$ given by (41) from the actual Dirichlet datum $d(s)=\cos \left(\frac{\pi}{2} s\right), l=2$ and $\beta=1$, evaluated at the side of the hexagon.

Furthermore, we depict the latter contribution for the different values of $\beta=\frac{1}{4}, \frac{1}{2}, 1,2,4$, where it is clearly shown that the error decreases drastically with the increase of $\beta$; see Figure 7 . We observe exponential decay for $z \neq z_{j}, j=1, \ldots, 6$ : in Figure 8 we depict the deviation from the actual Dirichlet data for three points on side (1) of the hexagon, namely $\alpha_{1}=(\sqrt{3}, 0), \alpha_{2}=\left(\sqrt{3}, \frac{3}{10}\right), \alpha_{3}=\left(\sqrt{3}, \frac{9}{10}\right)$, with $\beta$ in the intervals $I_{1}=[1,8], I_{2}=[1,10], I_{3}=[1,58]$, respectively. 


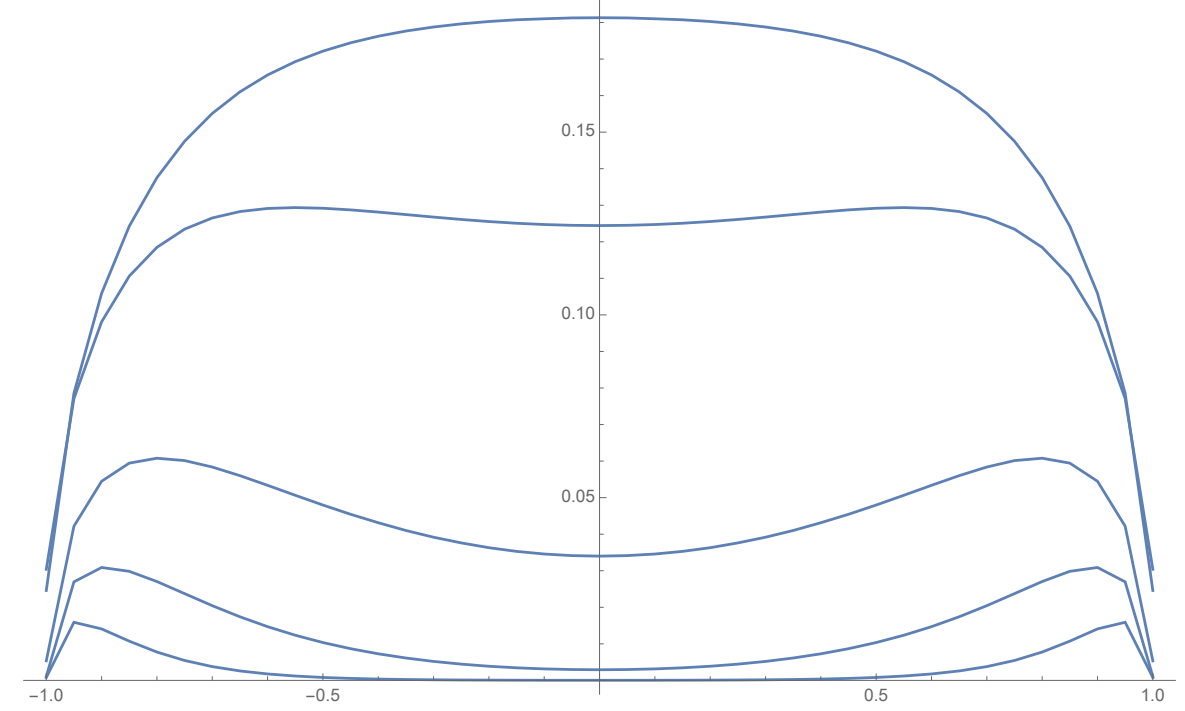

Figure 7. The deviation of the known part of the solution $q$ given by (41) from the actual Dirichlet datum $d(s)=\cos \left(\frac{\pi}{2} s\right)$ and $l=2$, evaluated at the side of the hexagon. This deviation is depicted for the different values of $\beta=\frac{1}{4}, \frac{1}{2}, 1,2,4$, and it decreases drastically with the increase of $\beta$.

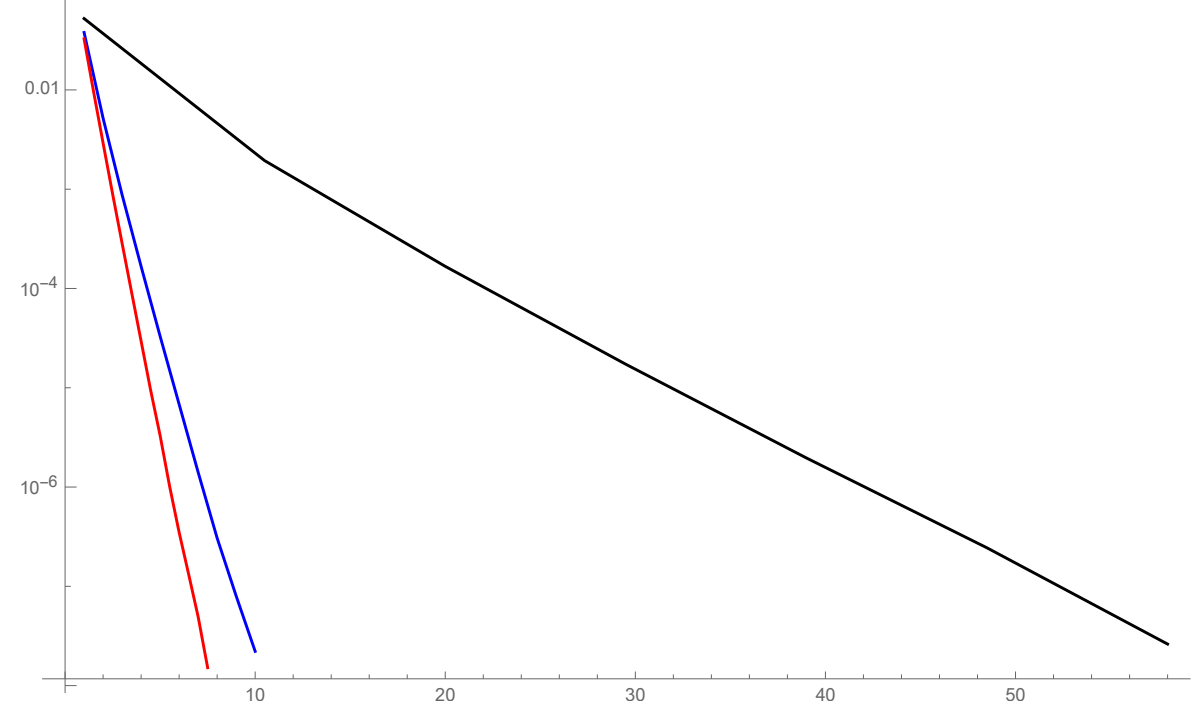

Figure 8. The deviation of the known part of the solution $q$ given by (41) from the actual Dirichlet datum $d(s)$, evaluated at three points of side (1) of the hexagon, namely $\alpha_{1}=(\sqrt{3}, 0)$ in red, $\alpha_{2}=\left(\sqrt{3}, \frac{3}{10}\right)$ in blue, $\alpha_{3}=\left(\sqrt{3}, \frac{9}{10}\right)$ in black. The deviation is depicted against $\beta$ and it displays exponential decay.

\section{Conclusions}

In this work we have presented the explicit solution of a particular boundary value problem for the modified Helmholtz equation in a regular hexagon: we have solved the case where the same Dirichlet datum $d(s)$ is prescribed in all sides of the hexagon, and this function is odd. This explicit solution is described in Proposition 1. We have also obtained an approximate analytical representation for the solution for the case that $d(s)$ is even. The exact representation is given by Equation (37), where the terms $F_{j}$ and $A_{j}$ are given in terms of $d(s)$, but the terms $B_{j}$ involve the unknown Neumann boundary value. However, these terms are exponentially small as $\beta \rightarrow \infty$. Thus, for the case of large $\beta$, Equation (37) provides the solution to this problem with an exponentially small error. The above analytical results were verified numerically in Section 5 . The rigorous investigation on the analytical and numerical accuracy of the latter approximate formula will be presented in future work. 
It should be noted that the arbitrary Dirichlet problem can be decomposed into 6 separate and simpler Dirichlet BVPs, which are defined in Section 2.3; the first of these BVPs is the symmetric Dirichlet problem. The analysis of the remaining problems is a work in progress.

Author Contributions: Conceptualization, K.K. and A.S.F.; methodology, K.K. and A.S.F.; validation, K.K. and A.S.F.; formal analysis, K.K. and A.S.F.; investigation, K.K. and A.S.F.; writing-original draft preparation, K.K. and A.S.F.; writing-review and editing, K.K. and A.S.F.; visualization, K.K. and A.S.F. All authors have read and agreed to the published version of the manuscript.

Funding: A.S.F. was supported by EPSRC, UK, via a senior fellowship.

Conflicts of Interest: The authors declare no conflict of interest.

\section{References}

1. Fokas, A.S. A unified transform method for solving linear and certain nonlinear PDEs. Proc. R. Soc. Lond. A Math. Phys. Eng. Sci. 1997, 453, 1411-1443. [CrossRef]

2. Fokas, A.S. On the integrability of linear and nonlinear partial differential equations. J. Math. Phys. 2000, 41, 4188-4237. [CrossRef]

3. Fokas, A.S. A new transform method for evolution partial differential equations. IMA J. Appl. Math. 2002, 67, 559-590. [CrossRef]

4. Biondini, G.; Wang, D. Initial-boundary-value problems for discrete linear evolution equations. IMA J. Appl. Math. 2010, 75, 968-997. [CrossRef]

5. Deconinck, B.; Trogdon, T.; Vasan, V. The method of Fokas for solving linear partial differential equations. SIAM Rev. 2014, 56, 159-186. [CrossRef]

6. Fokas, A.S. A Unified Approach to Boundary Value Problems; SIAM: Garden Grove, CA, USA, 2008.

7. Pelloni, B. The spectral representation of two-point boundary-value problems for third-order linear evolution partial differential equations. Proc. R. Soc. Lond. A Math. Phys. Eng. Sci. 2005, 461, 2965-2984. [CrossRef]

8. Pelloni, B.; Smith, D.A. Spectral theory of some non-selfadjoint linear differential operators. Proc. R. Soc. A Math. Phys. Eng. Sci. 2013, 469, 20130019. [CrossRef]

9. Pelloni, B.; Smith, D.A. Evolution PDEs and augmented eigenfunctions. Half-line. J. Spectr. Theory 2016, 6, 185-213. [CrossRef]

10. Smith, D.A. Well-posed two-point initial-boundary value problems with arbitrary boundary conditions. In Mathematical Proceedings of the Cambridge Philosophical Society; Cambridge University Press: Cambridge, UK, 2012; Volume 152, pp. 473-496.

11. Fokas, A.S. Two-dimensional linear partial differential equations in a convex polygon. Proc. R. Soc. Lond. Ser. A Math. Phys. Eng. Sci. 2001, 457, 371-393. [CrossRef]

12. Kalimeris, K. INITIAL and Boundary Value Problems in Two and Three Dimensions. Ph.D. Thesis, University of Cambridge, Cambridge, UK, 2010.

13. Spence, E.A. Boundary Value Problems for Linear Elliptic PDEs. Ph.D. Thesis, University of Cambridge, Cambridge, UK, 2011.

14. Batal, A.; Fokas, A.S.; Özsari, T. Uniform transform method for boundary value problems involving mixed derivatives. arXiv 2020, arXiv:2002.01057.

15. Fokas, A.S.; Himonas, A.A.; Mantzavinos, D. The nonlinear Schrödinger equation on the half-line. Trans. Am. Math. Soc. 2017, 369, 681-709. [CrossRef]

16. Himonas, A.A.; Mantzavinos, D. On the Initial-Boundary Value Problem for the Linearized Boussinesq Equation. Stud. Appl. Math. 2015, 134, 62-100. [CrossRef]

17. Himonas, A.A.; Mantzavinos, D.; Yan, F. Initial-boundary value problems for a reaction-diffusion equation. J. Math. Phys. 2019, 60, 081509. [CrossRef]

18. Özsari, T.; Yolcu, N. The initial-boundary value problem for the biharmonic Schrödinger equation on the half-line. Commun. Pure Appl. Anal. 2019, 18, 3285-3316. [CrossRef]

19. Kalimeris, K.; Özsarı, T. An elementary proof of the lack of null controllability for the heat equation on the half line. Appl. Math. Lett. 2020, 104, 106241. [CrossRef]

20. Crowdy, D.G.; Davis, A.M. Stokes flow singularities in a two-dimensional channel: A novel transform approach with application to microswimming. Proc. R. Soc. A 2013, 469, 20130198. 
21. Deconinck, B.; Oliveras, K. The instability of periodic surface gravity waves. J. Fluid Mech. 2011, 675, $141-167$. [CrossRef]

22. Fokas, A.S.; Kalimeris, K. Water waves with moving boundaries. J. Fluid Mech. 2017, 832, 641-665. [CrossRef]

23. Oliveras, K. Stability of Periodic Surface Gravity Water Waves. Ph.D. Thesis, University of Washington, Seattle, WA, USA, 2009.

24. Plümacher, D.; Oberlack, M.; Wang, Y.; Smuda, M. On a non-linear droplet oscillation theory via the unified method. Phys. Fluids 2020, 32, 067104. [CrossRef]

25. Vasan, V.; Deconinck, B. The inverse water wave problem of bathymetry detection. J. Fluid Mech. 2013, 714, 562-590. [CrossRef]

26. Ablowitz, M.J.; Fokas, A.S.; Musslimani, Z.H. On a new non-local formulation of water waves. J. Fluid Mech. 2006, 562, 313-343. [CrossRef]

27. Nicholls, D. A high-order perturbation of surfaces (HOPS) approach to Fokas integral equations: Three-dimensional layered-media scattering. Q. Appl. Math. 2016, 74, 61-87. [CrossRef]

28. Ashton, A.C.L. Laplace's equation on convex polyhedra via the unified method. Proc. R. Soc. A Math. Phys. Eng. Sci. 2015, 471, 20140884. [CrossRef]

29. Crowdy, D. A transform method for Laplace's equation in multiply connected circular domains. IMA J. Appl. Math. 2015, 80, 1902-1931. [CrossRef]

30. Dassios, G.; Fokas, A.S. The basic elliptic equations in an equilateral triangle. Proc. R. Soc. A Math. Phys. Eng. Sci. 2005, 461, 2721-2748. [CrossRef]

31. Fokas, A.S.; Kapaev, A.A. A Riemann-Hilbert approach to the Laplace equation. J. Math. Anal. Appl. 2000, 251, 770-804. [CrossRef]

32. Fokas, A.S.; Kapaev, A.A. On a transform method for the Laplace equation in a polygon. IMA J. Appl. Math. 2003, 68, 355-408. [CrossRef]

33. Luca, E.; Crowdy, D.G. A transform method for the biharmonic equation in multiply connected circular domains. IMA J. Appl. Math. 2018, 83, 942-976. [CrossRef]

34. Antipov, Y.A.; Fokas, A.S. The modified Helmholtz equation in a semi-strip. In Mathematical Proceedings of the Cambridge Philosophical Society; Cambridge University Press: Cambridge, UK, 2005; Volume 138, pp. 339-365.

35. Ashton, A.C.L. On the rigorous foundations of the Fokas method for linear elliptic partial differential equations. Proc. R. Soc. A Math. Phys. Eng. Sci. 2012, 468, 1325-1331. [CrossRef]

36. Ashton, A.C.L. The Spectral Dirichlet-Neumann Map for Laplace's Equation in a Convex Polygon. SIAM J. Math. Anal. 2013, 45, 3575-3591. [CrossRef]

37. Colbrook, M.J. Extending the unified transform: Curvilinear polygons and variable coefficient PDEs. IMA J. Numer. Anal. 2020, 40, 976-1004. [CrossRef]

38. Colbrook, M.J.; Flyer, N.; Fornberg, B. On the Fokas method for the solution of elliptic problems in both convex and non-convex polygonal domains. J. Comput. Phys. 2018, 374, 996-1016. [CrossRef]

39. Colbrook, M.J.; Fokas, A.S. Computing eigenvalues and eigenfunctions of the Laplacian for convex polygons. Appl. Numer. Math. 2018, 126, 1-17. [CrossRef]

40. Colbrook, M.J.; Fokas, A.S.; Hashemzadeh, P. A Hybrid Analytical-Numerical Technique for Elliptic PDEs. SIAM J. Sci. Comput. 2019, 41, A1066-A1090. [CrossRef]

41. de Barros, F.P.J.; Colbrook, M.J.; Fokas, A.S. A hybrid analytical-numerical method for solving advection-dispersion problems on a half-line. Int. J. Heat Mass Transf. 2019, 139, 482-491. [CrossRef]

42. Davis, C.I.R.; Fornberg, B. A spectrally accurate numerical implementation of the Fokas transform method for Helmholtz-type PDEs. Complex Var. Elliptic Equ. 2014, 59, 564-577. [CrossRef]

43. Fokas, A.S.; Nachbin, A. Water waves over a variable bottom: A non-local formulation and conformal mappings. J. Fluid Mech. 2012, 695, 288-309. [CrossRef]

44. Fornberg, B.; Flyer, N. A numerical implementation of Fokas boundary integral approach: Laplace's equation on a polygonal domain. Proc. R. Soc. A Math. Phys. Eng. Sci. 2011, 467, 2983-3003. [CrossRef]

45. Grylonakis, E.N.G.; Filelis-Papadopoulos, C.K.; Gravvanis, G.A. A class of unified transform techniques for solving linear elliptic PDEs in convex polygons. Appl. Numer. Math. 2018, 129, 159-180. [CrossRef]

46. Hashemzadeh, P.; Fokas, A.S.; Smitheman, S.A. A numerical technique for linear elliptic partial differential equations in polygonal domains. Proc. Math. Phys. Eng. Sci. 2015, 471, 20140747. [CrossRef]

47. Trogdon, T.; Biondini, G. Evolution partial differential equations with discontinuous data. Q. Appl. Math. 2019, 77, 689-726. [CrossRef] 
48. Lamé, G. Mémoire sur la propagation de la chaleur dans les polyèdres, et principalement dans le prisme triangulaire régulier. J. I'Ecole Poly Tech. 1833, 22, 194-251.

49. Fokas, A.S.; Kalimeris, K. Eigenvalues for the Laplace operator in the interior of an equilateral triangle. Comput. Methods Funct. Theory 2014, 14, 1-33. [CrossRef]

50. Pinsky, M.A. The eigenvalues of an equilateral triangle. SIAM J. Math. Anal. 1980, 11, 819-827. [CrossRef]

51. Pinsky, M.A. Completeness of the eigenfunctions of the equilateral triangle. SIAM J. Math. Anal. 1985, 16, 848-851. [CrossRef]

52. Práger, M. Eigenvalues and eigenfunctions of the Laplace operator on an equilateral triangle. Appl. Math. 1998, 43, 311-320. [CrossRef]

53. Terras, R.; Swanson, R. Image methods for constructing Green's functions and eigenfunctions for domains with plane boundaries. J. Math. Phys. 1980, 21, 2140-2153. [CrossRef]

54. McCartin, B.J. Eigenstructure of the equilateral triangle, Part I: The Dirichlet problem. Siam Rev. 2003, 45, 267-287. [CrossRef]

55. McCartin, B.J. Eigenstructure of the equilateral triangle, Part II: The Neumann problem. Math. Probl. Eng. 2002, 8, doi:10.1080/1024123021000053664. [CrossRef]

56. McCartin, B.J. Laplacian Eigenstructure of the Equilateral Triangle; Hikari Limited: Rousse, Bulgaria, 2011.

(C) 2020 by the authors. Licensee MDPI, Basel, Switzerland. This article is an open access article distributed under the terms and conditions of the Creative Commons Attribution (CC BY) license (http://creativecommons.org/licenses/by/4.0/). 